\title{
Extreme hyperferritinemia: etiological spectrum and impact on prognosis
}

\author{
N. Belfeki ${ }^{1}$, A. Strazzulla1 ${ }^{1}$, M. Picque ${ }^{2}$, S. Diamantis ${ }^{1}$ \\ ${ }^{1}$ Department of Internal Medicine, Groupe Hospitalier Sud lle de France, Melun, France; \\ 2Laboratory Department, Groupe Hospitalier Sud Ile de France, Melun, France
}

\begin{abstract}
SUMMARY
Hyperferritinemia can be the result of inflammation, infection, iron overload, or other uncommon pathologies including hemophagocytic lymphohistiocytosis (HLH). The significance of its elevation and its association with poor prognosis and critical clinical situations is unclear.

To study the spectrum of diagnosis associated with elevated serum ferritin, we made a retrospective review of patients admitted to our center from 2015 and 2017 with serum ferritin levels above $2000 \mu \mathrm{g} \mathrm{L}{ }^{-1}$. The $\mathrm{H}$ score was retrospectively assessed in all cases to evaluate the possible presence of HLH. The degree of ferritinemia found was compared with the evaluation of the undelying diagnosis and the results of laboratory examinations. A total of 77 patients were identified with a serum ferritin level $>2000 \mu \mathrm{g}$ L-1. Hematological malignancy was the most prevalent diagnosis with $n=20$; severe infection was next with $n=14$. Eleven patients were diagnosed with HLH. The hemophagocytosis pictures on bone marrow smear and mortality rate were significantly correlated with ferritin level above $6000 \mu \mathrm{g} \mathrm{L}^{-1}(\mathrm{p}<0.01)$. The comparison of the HLH subgroup with the rest of the cohort showed that fever, cytopenia (anemia, leucopenia, neutropenia and thrombocytopenia), hemophagocytosis pictures, and major liver disturbances were significantly increased in the HLH subgroup. The H score was significantly elevated in the subgroup of patients with ferritin above $6000 \mu \mathrm{g} \mathrm{L} \mathrm{L}^{-1}$, with a significatively higher probability of HLH $(\mathrm{p}<0.01)$. The mortality rate at 3 months was significantly increased in the HLH subgroup. Extreme hyperferritin cannot be considered as a specific marker for the diagnosis. The cut off of $6000 \mu \mathrm{g} \mathrm{L}^{-1}$ is significantly associated with HLH diagnosis. The $\mathrm{H}$ score is an interesting screening tool that physicians should use to rule out the probability of HLH when facing critical clinical situations.
\end{abstract}

Key words: Ferritin; inflammation; HLH; mortality.

Reumatismo, 2019; 71 (4): 199-202

\section{INTRODUCTION}

1 erritin is found in most tissues as a cytosolic protein and in small amounts secreted into the serum. It plays a key role in the storage of iron in soluble and nontoxic form and its release (1). If ferritin is high, there is iron in excess or, alternative$1 y$, there is an acute inflammatory reaction in which ferritin is mobilized without iron excess. It can be elevated in multiple conditions including inflammation, infection and malignancy.

The importance of this abnormal value is not well understood and its association with poor prognosis and critical clinical situations is unclear. Various studies suggested that markedly elevated ferritin in hospitalized patients is often associated with hemophagocytic lymphohistiocytosis syndrome (HLH) but cannot be considered as a specific marker $(2,3)$. HLH syndrome is a rare diagnosis that is difficult to distinguish from other diseases because it has protean clinical, biological and histological presentation. The $\mathrm{H}$ score is a composite diagnostic score that estimates an individual's risk of having HLH (4). To understand the significance of elevated ferritin in hospitalized patients and to determine the underlying conditions, we performed a retrospective analysis of patients admitted to our hospital and found to have ferritin level above $2000 \mu \mathrm{g} \mathrm{\textrm {L } ^ { - 1 }}$. Furthermore, we assessed retrospectively the diagnostic score for HLH for all patients to demonstrate the difficulties of managing situations that can mimic HLH clinically. $\overline{\text { Corresponding author: }}$ Nabil Belfeki

Department of Internal Medicine, Groupe Hospitalier Sud lle de France, 270 Avenue Marc Jacquet,

77000 Melun, France

E-mail: belfeki.nabil@gmail.com 


\section{PATIENTS AND METHODS}

We recruited retrospectively all hospitalized patients who had at least one serum ferritin measurement above $2000 \mu \mathrm{g} \mathrm{L}^{-1}$ between 2015 and 2017 at Groupe Hospitalier Sud Ile de France, Melun, France. Demographic data, etiological spectrum, and prognosis were gathered from medical records. In each patient, the highest level of ferritin was retained. Patients were divided according to the etiologies as having HLH, inflammatory diseases, hematologic malignancy, renal failure and other. HLH was diagnosed according to hemophagocytic lymphohistiocytosis (HLH)-2004 criteria by the Histiocyte Society (5). Inflammatory disease, solid tumor, and hematological malignancy were defined as histological evidence. HIV and non-HIV infections were confirmed with positive serologies. Liver disease was defined as established diagnosis of chronic liver disease and renal failure was defined as having replacement therapy or having a glomerular filtration rate lower than $20 \mathrm{~mL} \mathrm{~min}^{-1} 1.73 \mathrm{~m}^{-2}$. Iron overload was considered in patients receiving regular red blood cell transfusions more than once a month for longer than six months. The most likely cause of hyperferritinemia was decided on the basis of available clinical data. In patients having more than one possible etiology, a single predominant underlying cause was decided. Patients were categorized into two groups according to serum ferritin level $>2000 \mu \mathrm{g} \mathrm{L}^{-1}$ and $<6000 \mathrm{mg} \mathrm{L}^{-1},>6000 \mu \mathrm{g} \mathrm{L}^{-1}$ and compared according to clinical features and routine laboratory parameters (cell blood count, fibrinogen, transaminases, triglycerides). The $\mathrm{H}$ score was retrospectively assessed in all cases to evaluate the probability of having HLH. Statistical analyses were performed using SPSS Statistics, version 20.0.

\section{RESULTS}

Seventy-seven patients who had at least one ferritin measurement above $2000 \mu \mathrm{g}$ $\mathrm{L}^{-1}$ were included. The mean duration of follow up was 24 months. The average age of these patients was 66 years (with extremes of 4 and 94 years). Of these patients, $57 \%$ were male. Median serum ferritin level was $3438 \mu \mathrm{g} \mathrm{L}^{-1}$ (interquartile range: $2300-193,370 \mu \mathrm{g} \mathrm{L}^{-1}$ ). Serum ferritin values were stratified as described in the method. $78.7 \%$ of the patients had serum ferritin levels 2000-6000 $\mu \mathrm{g} \mathrm{L}^{-1}$ and $21.3 \%$ had serum ferritin levels above $6000 \mu \mathrm{g} \mathrm{L} \mathrm{L}^{-1}$. The most common diagnosis category was hematological malignancy $(n=20,23.7 \%)$ followed by severe infection $(n=14,19 \%)$, chronic kidney disease and iron overload syndromes ( $\mathrm{n}=13,18 \%$ each), and HLH ( $\mathrm{n}$ $=11,14 \%$ ). There were relatively few cases of hepato-cellular disease $(\mathrm{n}=5,6 \%)$, and adult onset Still disease $(\mathrm{n}=1,1.3 \%)$. The Table I summarizes comparative demographic data and the etiological spectrum of our cohort according to ferritin levels. The spectrum of clinical features and laboratory findings according to the level of ferritin is represented in the Table II. The hemophagocytosis pictures on bone marrow smear and mortality rate were significantly

Table I - Epidemiological data and etiological spectrum according to ferritin levels.

\begin{tabular}{|l|c|c|c|}
\hline & $\mathbf{2 0 0 0}<$ Ferritin $<6000 \mathbf{~ g ~ L}^{-1}$ & Ferritin $>6000 \mu \mathbf{g ~ L}^{-1}$ & Total \\
\hline Patients (n, \%) & $61(79)$ & $16(21)$ & $77(100)$ \\
\hline Sex ratio (M/F) & $34 / 27$ & $10 / 6$ & $44 / 33$ \\
\hline Median age & 75 & 56 & 66 \\
\hline HLH $(n, \%)$ & $1(10)$ & $10(90)$ & $11(100)$ \\
\hline Chronic liver disease $(n, \%)$ & $5(100)$ & $0(0)$ & $5(100)$ \\
\hline Severe infection (n, \%) & $11(80)$ & $3(20)$ & $14(100)$ \\
\hline Chronic kidney disease $(n, \%)$ & $13(100)$ & 0 & $13(100)$ \\
\hline Hematological malignancy (n, \%) & $13(65)$ & $7(35)$ & $20(100)$ \\
\hline Iron overload $(n, \%)$ & $13(93)$ & $0(0)$ & $14(100)$ \\
\hline
\end{tabular}


Table II - Comparison of clinical presentation and laboratory findings according to ferritin levels.

\begin{tabular}{|l|c|c|}
\hline & $\mathbf{2 0 0 0}<$ Ferritin $<6000 \mathbf{~ g ~ L}^{-1}$ & Ferritin $>\mathbf{6 0 0 0} \mathbf{~ g ~ L}^{-1}$ \\
\hline Hepatomegaly (n, \%) & $6(75)$ & $2(25)$ \\
\hline Splenomegaly (n, \%) & $13(72.2)$ & $5(27.8)$ \\
\hline Adenomegaly (n, \%) & $15(68.2 \%)$ & $7(31.8 \%)$ \\
\hline Fever (n, \%) & $23(54)$ & $19(46)$ \\
\hline Hemoglobin (G/dL) & 8.2 & 7.0 \\
\hline Leukocytes (G/L) & 7.8 & 3.5 \\
\hline Neutrophils(G/L) & 4.5 & 1.5 \\
\hline Platelets (G/L) & 132 & 36 \\
\hline Triglycerides (g/L) & 1.30 & 1.29 \\
\hline Fibrinogen (g/L) & 4.12 & 3.75 \\
\hline AST/ALT (UI/L) & $53 / 43$ & $450 / 400$ \\
\hline H score & 87 & 235 \\
\hline Probability of HLH (\%) & 0.90 & 98 \\
\hline
\end{tabular}

correlated with ferritin level above $6000 \mu \mathrm{g}$ $\mathrm{L}^{-1}(\mathrm{p}<0.01)$. The comparison of the HLH subgroup with the rest of the cohort showed that fever, cytopenia (anemia, leucopenia, neutropenia and thrombocytopenia), hemophagocytosis pictures, and major liver disturbances significantly increased in the HLH subgroup. Likewise, the 3-month mortality rate significantly increased in the HLH subgroup. The Table III illustrate a comparison of demographic and clinical data in HLH and non HLH patients.

\section{DISCUSSION \\ AND CONCLUSIONS}

Ferritin is an intracellular protein that stores iron. Plasma ferritin is an indirect marker of the total amount of iron stored in the body, hence serum ferritin is used as a diagnostic test. Elevated levels of ferritin indicate iron overload, but they are not specific as ferritin is an acute protein that increases in response to inflammatory states including malignancy, infection and in liver or renal diseases. Rheumatologists are often asked to manage patients with this condition because they are often thought to be exclusive. This situation is protean and its spectrum is wide. Previously published cohort studies choose $1000 \mu \mathrm{g} \mathrm{L}^{-1}$ as an appropriate cut off value for markedly elevated ferritin (2). Other studies suggested that this level sets the threshold too low and that the sensitivity and specificity of ferritin for HLH should be raised to a cutoff level above $2000 \mu \mathrm{g}$ $\mathrm{L}^{-1}$ (3). This data is considered by other authors who used the same cut off level to

Table III - Comparison of demographic and clinical data in patients with and without the diagnosis of $\mathrm{HLH}$ syndrome.

\begin{tabular}{|l|c|c|c|}
\hline & HLH patients & Non HLH patients & P \\
\hline Sex ratio M/F & $9 / 2$ & $37 / 29$ & \\
\hline Median age (years) & 55 & 67 & 0.27 \\
\hline Hepatomegaly (n) & 1 & 7 & NS \\
\hline Splenomegaly $(\mathrm{n})$ & 4 & 14 & $\mathrm{NS}$ \\
\hline Adenomegaly $(\mathrm{n})$ & 6 & 16 & $\mathrm{NS}$ \\
\hline Hemophagocytosis (n) & 9 & 1 & $<0.01$ \\
\hline Median ferritinemia $(\mu \mathrm{g} \mathrm{L}-1)$ & 39892 & 3438 & $<0.01$ \\
\hline Fever $(\mathrm{n})$ & 11 & 31 & $<0.01$ \\
\hline Median hemoglobin (g/dl) & $6.5 \pm 1.7$ & $8.6 \pm 2.0$ & 0.02 \\
\hline Leukocyte (G/L) & $3.5 \pm 2$ & $8.2 \pm 5.6$ & 0.04 \\
\hline Neutrophils (G/L) & $1.8 \pm 1.5$ & $5.8 \pm 4.5$ & $<0.01$ \\
\hline Platelets & $57 \pm 39$ & $153 \pm 125$ & 0.045 \\
\hline Triglycerides (g/L) & 1.29 & 4.0 & $\mathrm{NS}$ \\
\hline Fibrinogen (g/L) & 2.68 & 4.0 & $\mathrm{NS}$ \\
\hline AST (Ul/L) & 3921 & 213 & $<0.01$ \\
\hline ALT (UI/L) & 466 & 145 & $<0.01$ \\
\hline H score & 273 & 89 & $<0.01$ \\
\hline Probability of HLH (\%) & 98 & 16 & $<0.01$ \\
\hline Three-month mortality $(\mathrm{n})$ & 9 & 11 & $<0.01$ \\
\hline
\end{tabular}


elaborate a diagnostic composite score for HLH called H score (4). In our cohort, we considered this cut off to try to elaborate the etiological spectrum of patients with markedly elevated ferritin and estimate the probability of developing HLH. On the basis of our results in an inpatient setting, the most likely causes for an elevated ferritin are hematological malignancy, severe infection, iron overload, and chronic kidney disease. HLH was diagnosed in $14 \%$ of cases. These findings are different from literature data where liver injury and infections seems to be most predominant etiologies. This could be explained by recruitment bias. In fact, our center is a general hospital with local recruitment, compared to other cohorts from tertiary teaching hospitals or reference centers for oncology or liver disease. Nevertheless, we recorded more cases of HLH (3). In the subgroup of patients with ferritin above $6000 \mu \mathrm{gL}^{-1}$, we recorded $90 \%$ of our HLH patients, suggesting that a higher threshold of serum ferritin would be more specific of HLH. In this subgroup, however, we reported patients with hematological malignancy $(35 \%)$ and sepsis $(20 \%)$ as well. We conclude that high ferritin level cannot be used as a screening tool for HLH diagnosis and should be integrated into the clinical picture of HLH including fever, cytopenia, and organomegalia. We assessed the $\mathrm{H}$ score in all patients and showed that the probability of developing HLH was superior to $90 \%$ in $17.3 \%$ of cases. Thus, there were probably 2 HLH patients misdiagnosed in our cohort. We recommend the use of $\mathrm{H}$ score in each clinical situation with ferritin above $6000 \mu^{-1} l^{-1}$ to help physicians not misdiagnose HLH. Markedly elevated ferritin levels are far more likely to accompany non-rheumatologic diseases like infection, hematological malignancy and iron overload. This study demonstrated that high ferritin could be considered as a factor of poor prognosis and high level of mortality in patients with a level above $6000 \mu \mathrm{g} \mathrm{L}^{-1}$. Further larger studies are certainly needed to confirm this finding. In accordance with previously published data, our findings support the fact that having high cut off value of ferritin, as used in the $\mathrm{H}$ score, may predict more patients with HLH diagnosis $(3,6)$. The measurement can help support but not confirm this diagnosis. Despite the limitations inherent in a retrospective review, our cohort did not suffer from recruitment bias in comparison with those reported in tertiary academic hospitals where patients are referred for liver transplantation (2).

\section{REFERENCES}

1. Meyron-Holtz EG, Moshe-Belizowski S, Cohen LA. A possible role for secreted ferritin in tissue iron distribution. J Neural Transm. 2011; 118: 337-47.

2. Moore C Jr, Ormseth M, Fuchs H. Causes and significance of markedly elevated serum ferritin levels in an academic medical center. $\mathbf{J}$ Clin Rheumatol. 2013; 19: 324-8.

3. Schram AM, Campigotto F, Mullally A, et al. Marked hyperferritinemia does not predict for HLH in the adult population. Blood. 2015; 125: $1548-52$.

4. Fardet L, Galicier L, Lambotte O, et al. Development and validation of the $\mathrm{H}$ Score, a score for the diagnosis of reactive hemophagocytic syndrome. Arthritis Rheumatol. 2014; 66: 2613-20.

5. Henter II, Horne AC, Aricó M, et al. HLH2004: diagnostic and therapeutic guidelines for hemophagocytic lymphohistiocytosis. Pediatr Blood Cancer. 2007; 48: 124-31.

6. Ota T. Hyperferritinemia and diseases. J UOEHD. 2000; 22: 189-200. 